\section{Sleeping Poorly ... a and Maybe Depressed: Comorbidity in Patients Referred for Formal Sleep Studies}

To the Editor: Disorders of sleep and wakefulness are estimated to affect 60 million Americans. ${ }^{1,2}$ Poor sleep, perhaps because it accompanies so many medical and psychiatric conditions, is likely to be present in $40 \%$ of adult patients that present to family medicine clinics, placing it among the most frequent issues that family doctors encounter. ${ }^{3}$

Two of the most common sleep disorders, insomnia and obstructive sleep apnea syndrome (OSAS), are often accompanied by depression ${ }^{4,5}$ although the reason for this is not well understood. Sleep disorders and mood disorders have many symptoms in common such as hypersomnolence, low energy, and irritability, which raises the possibility of diagnostic confusion between them.

The objectives of this study were to examine the prevalence of mood disorders in people with sleep complaints and to learn whether primary sleep disorders might sometimes masquerade as mood disorders.

\section{Methods}

We administered a 7-item questionnaire to 100 consecutive patients referred to our sleep center for polysomnography (Table 1). We inquired about previous diagnosis of depression and, when it was present, about patterns of evaluation and treatment for it. The final item was an open-ended question to elicit the patient's own perceptions of how their mood and sleep complaints are related.

\section{Findings}

Of the 100 patients, 53 respondents (53\%) carried a diagnosis of depression in their past medical history. The following data relates to these 53 people: $37(70 \%)$ of 53 people were actively taking antidepressant medication at the time of their referral to the sleep lab.

\section{Funding: none.}

Conflict of interest: The author served briefly on the speakers bureau for Sepracor, the manufacturer of Lunesta, in 2005.

\section{Table 1. The Sleep and Mood Questionnaire}

Q1. Have you ever been diagnosed with Depression? If "yes," please continue.

Q2. Have you seen a health care professional within the past 6 months for depression?

If yes, was this a family doctor, psychiatrist, therapist, or other health care professional?

Q3. Have you ever used a medication to treat your depression?

If yes, what medication(s) have you tried?

Q4. Do you currently take antidepressant medications?

Q5. Have you ever tried medications specifically to improve your sleep?

If yes, what medication(s) have you tried?

Q6. Who referred you for your overnight sleep study?

Q7. Would you like to comment on the relationship between your sleep and your depression?

Regarding referral patterns, we found that $21(40 \%)$ of the 53 respondents had been referred to the sleep center by their primary care provider; 17 (32\%) of the 53 respondents had been referred by subspecialists, and 13 (24\%) of the 53 respondents had been self-referred (and 2 [4\%] of 53 respondents did not respond to this question).

Eleven patients commented on the relationship between their depression and their sleep complaint (Table 2). Six of them (patient nos. 10, 23, 24, 29, 40 , and 53) clearly believed that their sleep disorder was primary and had triggered their depressed mood. Furthermore, some of their comments seem to dispute the diagnosis of depression entirely; they assert instead that their physician had wrongly attributed symptoms of insomnia (patient no. 29), low energy (patient no. 23), and poor motivation (patient no. 40) to depression rather than to a sleep disorder.

\section{Discussion}

In this survey of 100 patients referred to our sleep center, we found that a very high proportion $(53 \%)$ of them had also been diagnosed with depression. This is consistent with other estimates of comorbidity between depression, insomnia, and obstructive sleep apnea. ${ }^{6}$ This high concordance serves as a reminder that when primary care physicians diagnose depression, they should question their 
Table 2. Patient Comments About the Relationship of Their Own Depression and Sleep

\begin{tabular}{ll}
\hline Patient no. & \multicolumn{1}{c}{ Comments } \\
\hline 4 & My insomnia evolved from stressful life situations \\
10 & Sleep problems cause my depression \\
12 & My depression has led to insomnia \\
18 & Sadness, bad dreams, awakening and ruminating \\
20 & Pain medications cause depression and anxiety \\
& and lead to poor sleep \\
23 & Disrupted sleep causes me to have less energy \\
& and to want to sleep during the day \\
24 & If I am sleep deprived, then my depression is \\
& exacerbated \\
29 & $\begin{array}{l}\text { Anxiety has a part in my trouble falling asleep } \\
\text { My depression has not been consistent, but my } \\
\text { trouble falling asleep has been }\end{array}$ \\
53 & $\begin{array}{l}\text { My inability to get things done makes me have } \\
\text { less self-esteem leading to worse depression }\end{array}$ \\
& It's hard to stay positive without a full amount of \\
& energy
\end{tabular}

patient closely about sleep complaints, especially obstructive breathing patterns and insomnia.

One weakness of this study is that it relies solely on patients' own perceptions of their diagnosis without validation by a depression scale or chart review (we plan to add this to future studies). Nevertheless, the narrative comments of some patients, in which they question their depression label, raises the possibility that sleep disorders may sometimes masquerade as mood disorders and delay timely diagnosis and treatment. In this survey, primary care clinicians initiated the sleep lab referral in only $40 \%$ of the respondents who presented with a diagnosis of depression. This author, who is a sleep disorders specialist with a background in family medicine, has heard many patients referred for sleep consultation remark, "my doctor kept telling me that I was depressed . . but my real problem is that I can't sleep!”
When patients present, as they frequently do, with hypersomnolence, fatigue, irritability, low energy, or diminished cognitive function, the provider's antennae likely will be raised to diagnose and treat depression. These findings serve as a small reminder that they need to be aware that common primary sleep pathologies such as obstructive sleep apnea or insomnia may also account for these symptoms.

Adam J. Sorscher, MD Minnesota Regional Sleep Disorders Center

Minneapolis, MN

Department of Community and Family Medicine Dartmouth Medical School

Hanover, NH adam.sorscher@dartmouth.edu

The author wishes to thank the polysomnography technicians at the Minnesota Regional Sleep Disorders Center for their work in administering and collecting the sleep and mood surveys.

\section{References}

1. Colten HR, Altevogt B, editors. Sleep disorders and sleep deprivation: an unmet public health problem. Washington, D.C.: The National Academies Press; 2006.

2. Hossain JL, Shapiro CM. The prevalence, cost implications, and management of sleep disorders: an overview. Sleep Breath 2002;6(2):85-102.

3. Ancoli-Israel S, Roth T. Characteristics of insomnia in the United States: results of the 1991 National Sleep Foundation Survey. I. Sleep 1999;22 (Suppl 2):S347-53.

4. Aikens JE, Vanable PA, Tadimeti L, Caruana-Montaldo B, Mendelson WB. Differential rates of psychopathology symptoms in periodic limb movement disorder, obstructive sleep apnea, psychophysiological insomnia, and insomnia with psychiatric disorder. Sleep 1999;22(6):775-80.

5. Buysse DJ, Reynolds CF, 3rd, Kupfer DJ, et al. Clinical diagnoses in 216 insomnia patients using the international classification of sleep disorders (ICSD), DSM-IV and ICD-10 categories: a report from the APA/NIMH DSM-IV field trial. Sleep 1994;17(7):630-7.

6. Schwartz DJ, Kohler WC, Karatinos G. Symptoms of depression in individuals with obstructive sleep apnea may be amenable to treatment with continuous positive airway pressure. Chest 2005 Sep;128(3):1304-9. 